\title{
Gut microbial modulation in the treatment of chemotherapy-induced diarrhea with Shenzhu Capsule
}

Jing Wang ${ }^{1 \dagger}$, Wuwen Feng ${ }^{1 \dagger}$, Shiyang Zhang ${ }^{1}$, Lu Chen ${ }^{1}$, Fei Tang ${ }^{1}$, Yongcheng Sheng ${ }^{1}$, Hui Ao ${ }^{1,2^{*}}$ (I) and Cheng Peng ${ }^{1 *}$

\begin{abstract}
Background: Gut microbiota plays a crucial role in the treatment of gastrointestinal (Gl) diseases such as chemotherapy-induced diarrhea (CID). Shenzhu Capsule (SZC) is a Chinese herbal formula, which is composed of Renshen (rhizomes of Panax ginseng C. A. Mey.) and Baizhu (rhizomes of Atractylodes macrocephala Koidz.). Many Chinese traditional anti-diarrheal formulae that contain Renshen and Baizhu are capable of effectively alleviating CID. However, the efficacy in vivo and potential mechanism of SZC (the form of compatibility of Renshen and Baizhu) in the treatment of CID had not been elucidated. Here, this study aimed to investigate whether SZC exhibited the antidiarrheal activity, and whether gut microbiota was involved in the therapeutic effect of SZC on CID.

Methods: High performance liquid chromatography (HPLC), gas chromatography-mass spectrometer (GC-MS) and infrared spectroscopy (IR) analyses were used to characterize the extracted components in SZC. The mice were orally administrated with $\mathrm{SZC}$ in a preventive mode on the first 2 days of this experiment, and then intraperitoneally injected with 5-FU (40 mg/kg/d) for 6 days. SZC treatment lasted until the 3rd day after the end of 5-FU chemotherapy. We investigated the effects of SZC on body weights, diarrhea, thymus/spleen indexes, colonic tissues, and gut microbiota. Colonic histology was examined by hematoxylin-eosin (HE) staining. 16S rDNA Amplicon Sequencing was used to analyze the gut microbial structure from fecal samples.

Results: SZC significantly increased the body weights and thymus/spleen indexes, alleviated diarrhea, and reversed histopathological changes of colons. In addition, gut microbiota analysis revealed that the overall structure of gut microbiota in CID mice was disturbed, but reversed to the normal state after SZC treatment. At genus level, SZC significantly inhibited the growth of some potential pathogens associated with diarrhea, such as Clostridiumm, Bacteroides, Parabacteroides, Alloprevotella, Acinetobacter and Pseudomonas.
\end{abstract}

Conclusions: In our study, these data illustrated that SZC inhibited the growth of many potential pathogens during the alleviation of CID. Gut microbial modulation was associated with the anti-diarrheal activity of SZC.

Keywords: Gut microbiota, Chemotherapy-induced diarrhea, Herbal medicine, 5-fluorouracil

\footnotetext{
* Correspondence: aohui2005@126.com; pengchengcxy@126.com

† Jing Wang and Wuwen Feng contributed equally to this work.

'School of Pharmacy, Chengdu University of Traditional Chinese Medicine,

Chengdu 611137, China

Full list of author information is available at the end of the article
}

(c) The Author(s). 2019 Open Access This article is distributed under the terms of the Creative Commons Attribution 4.0 International License (http://creativecommons.org/licenses/by/4.0/), which permits unrestricted use, distribution, and reproduction in any medium, provided you give appropriate credit to the original author(s) and the source, provide a link to the Creative Commons license, and indicate if changes were made. The Creative Commons Public Domain Dedication waiver (http://creativecommons.org/publicdomain/zero/1.0/) applies to the data made available in this article, unless otherwise stated. 


\section{Background}

Gut microbiota, known as a complex ecological community colonized in human gastrointestinal (GI) tract, can interact with host factors to affect normal physiology and diseases [1]. Healthy gut microbiota contributes to the metabolism of nutrition and drugs, structural integrity of intestinal mucosal barrier, immune regulation, and inhibition of pathogens [2,3]. Gut microbiota in the disordered state is likely to trigger many diseases, such as diarrhea [4], inflammatory bowel disease [5], metabolism disturbances (obesity, insulin resistance and diabetes) [6], and cardiovascular diseases [7]. Due to its ubiquitous roles in the maintenance of gut homeostasis and human health, gut microbiota exhibits great effects on the development and progression of diseases $[8,9]$.

Among the diseases related to altered gut microbiota, chemotherapy-induced diarrhea (CID) is one of the most common digestive complications in cancer patients treated with chemotherapeutic drugs such as fluoropyrimidines and irinotecan [10]. CID has been found to occur in $50-80 \%$ of cancer patients, especially those with advanced cancer [11]. In addition, severe diarrhea (grade $3 / 4$ ) was reported in $16.6 \%$ colorectal cancer patients receiving 5-fluorouracil (5-FU), the first-line chemotherapeutic drug for colorectal cancer [12]. A growing number of evidences showed that CID could directly threaten the lives of patients, or indirectly affect the cycle and efficacy of chemotherapy [13]. Therefore, at present, medication is being introduced into the treatment of CID. Modern medicines such as loperamide and octreotide are often used to relieve CID in clinical practices [14]. However, their therapeutic effects are not satisfactory sometimes. For example, loperamide cannot alleviate several diarrheal features including abdominal cramps, an inability to eat, increasing fatigue, increasing weakness, chest pain, gastrointestinal bleeding [15]. Long-acting octreotide acetate was not effective in preventing or relieving diarrhea in cancer patients undergoing chemoradiation therapy [16]. In a word, there is still a lack of safe and effective modern medicines for the prevention and treatment of CID. Therefore, considerable attentions have been paid to complementary or alternative medicines such as traditional herbal medicines $[17,18]$.

Shenzhu Capsule (SZC), a formula of Chinese herbal medicine, is composed of ginsenosides and polysaccharides from Renshen (rhizomes of Panax ginseng C. A. Mey.), essential oil and polysaccharides from Baizhu (rhizomes of Atractylodes macrocephala Koidz.). In the past few years, our researches mainly focused on the therapeutic effects of SZC for gastrointestinal diseases. For example, we had reported that SZC induced apoptosis of gastric cancer cells through mitochondrial pathway [19-21]. In addition, SZC could reversely regulate the expression of some genes (metabolic, ion channels and transport proteins) in SGC-7901 tumor-bearing mice, resulting in the improvement of gastric cancer through multiple targets [22]. Moreover, SZC not only regulated immune and inflammatory responses during the treatment of ulcerative colitis (UC), but also acted on UC complications (bloody diarrhea) and prevented UC from developing into colorectal cancer [23]. At present, our attentions are being paid to investigate whether SZC exhibits therapeutic effect on CID, the GI side effect of chemotherapeutic drugs. As is known, many Chinese traditional anti-diarrheal formulae such as Shenling Baizhu Powder, Buzhong Yiqi Decoction and Sijunzi Decoction, all of which contain Renshen and Baizhu, had been proved to possess the ability of alleviating CID [24-26]. However, no study has determined if SZC (the form of compatibility of Renshen and Baizhu) exerts any therapeutic effect on CID. In recent years, gut microbiota has become an important frontier to reveal the therapeutic mechanisms of medicines [27]. Therefore, it is of great significance to investigate the therapeutic effect of SZC on CID based on gut microbiota.

In this study, we simulated a CID model in mice receiving 5-FU chemotherapy to evaluate the efficacy and interpret the potential mechanism of SZC in the treatment of diarrhea. Both diarrhea rates and histological changes of colonic tissues were recorded. $16 \mathrm{~S}$ rDNA Amplicon Sequencing was used to analyze the gut microbial structure from fecal samples.

\section{Methods}

\section{Chemicals and reagents}

5-FU injection $(25 \mathrm{mg} / \mathrm{mL}$, No. 1708061) was provided by Tianjin JinYao Pharmaceutical Co., Ltd. (Tianjin, China). Standard compounds (purity $>98 \%$ ) of ginsenosides Rg1, Rc, Rd, Re, Rb1, Rb2 and Rf were purchased from Chengdu Must Bio-Technology Co. Ltd. (Chengdu, China). Other chemicals and reagents were obtained from commercial sources.

\section{Experimental animals}

Twenty-four, 8-week-old SPF graded Kunming mice (12 males and 12 females) (Chengdu Dossy Experimental Animal Co., LTD., Chengdu, China, No. SCXK (chuan) 014-028) were adapted to the laboratory environment for 3 days before the experiment. The mice were housed under the standard environment (temperature of $25 \pm$ $2{ }^{\circ} \mathrm{C}$, humidity of $50 \pm 5 \%$, and a $12 \mathrm{~h}$ light $/ 12 \mathrm{~h}$ dark cycle) with free access to the same water and diets. All efforts were made to minimize animal suffering. All experimental procedures involving animals were agreed by Animal Ethics Committee (Approval No. AEC-201706) in Chengdu University of Traditional Chinese Medicine (CDUTCM) (Chengdu, China). 


\section{Collection of Renshen and Baizhu}

The materials were purchased from Lotus Pond Chinese herbal medicine market, Chengdu, Sichuan Province, People's Republic of China. They were respectively identified as the rhizomes of Panax ginseng C. A. Mey. and Atractylodes macrocephala Koidz. by Associate Professor Lu Chen from School of Pharmacy in CDUTCM. The voucher specimens of Renshen and Baizhu (No. 17102101 and 17102102, respectively) were deposited at School of Pharmacy in CDUTCM.

\section{Preparation and characterization of SZC}

SZC is composed of ginsenosides and polysaccharides isolated from Renshen, essential oil and polysaccharides isolated from Baizhu. In this study, we extracted and characterized those components according to the preparing process of SZC [24].

\section{Preparation and high performance liquid chromatography (HPLC) characterization of ginsenosides from Renshen in SZC}

Renshen powder $(200 \mathrm{~g})$ was refluxed 3 times by $60 \%$ ethanol $(8 \times)$ for total $6 \mathrm{~h}$. Then, the combined extracts were concentrated by vacuum-rotary evaporation. The concentrate was eluted by 70\% ethanol on D101 macroporous adsorptive resin. All the collected eluents were concentrated, and then dried at $40{ }^{\circ} \mathrm{C}$ in a vacuum oven. Final residue of ginsenosides $(1.4 \mathrm{mg})$ was dissolved in 1 $\mathrm{mL}$ methanol and then filtered through a $0.45 \mu \mathrm{m}$ filter membrane. Ginsenosides were characterized by HPLC (1260, Agilent, USA) with a C18 column $(250 \mathrm{~mm} \times 4.6$ $\mathrm{mm}$ i.d., particle size $5 \mu \mathrm{m}$ ) (Cosmosil, Japan). The column was eluted at $35^{\circ} \mathrm{C}$ with a detection wavelength at $203 \mathrm{~nm}$ and an injection volume of $10 \mu \mathrm{L}$. The flow rate of the mobile phase of water (A) and acetonitrile (B) was set at $1.0 \mathrm{~mL} / \mathrm{min}$. Gradient separation was based on the following: $0-10 \mathrm{~min}, 18-21 \% \mathrm{~B} ; 10-20 \mathrm{~min}, 21-22 \% \mathrm{~B}$; 20-30 min, $22-26 \%$ B; $30-40$ min, $26-30 \%$ B; $40-55$ min, $30-32 \%$ B; $55-75$ min, $32-33.8 \%$ B; $75-90 \mathrm{~min}$, $33.8-38 \%$ B; $90-105$ min, 38-45\% B; $105-110$ min, 45$18 \% \mathrm{~B}$. And the standard curves were obtained based on the absorbencies of different concentrations of standard ginsenosides Rg1, Re, Rf, Rc, Rd, Rb1 and Rb2.

Preparation and gas chromatography-mass spectrometer (GC-MS) characterization of essential oil from Baizhu in SZC

Baizhu powder $(200 \mathrm{~g})$ was refluxed by distilled water $(10 \times)$ for $12 \mathrm{~h}$. Final essential oil was gathered by steam distillation from the powder. Essential oil was characterized by GC-MS (GC 7890A and MS 5975C, Agilent, USA). Chromatographic separation was achieved on a capillary column HP-5MS $(30 \mathrm{~m} \times 0.25 \mathrm{~mm}$ i.d.; film thickness $0.25 \mu \mathrm{m}$ ). The electron ionization system operated at $70 \mathrm{eV}$ of ionization energy. Helium was used as the carrier gas. $1 \mu \mathrm{L}$ sample (essential oil: $\mathrm{n}$-hexane, 1 : 100 dilution) was injected and the injector temperature was set at $230.0^{\circ} \mathrm{C}$. Initial oven temperature was set at $100.0^{\circ} \mathrm{C}$, then increased to $120.0^{\circ} \mathrm{C}$ at the rate of $3.0^{\circ} \mathrm{C} /$ min and held at $120.0^{\circ} \mathrm{C}$ for $20 \mathrm{~min}$, and finally increased to $220.0^{\circ} \mathrm{C}$ at the rate of $3.0^{\circ} \mathrm{C} / \mathrm{min}$. MS was recorded in the range of $35-550 \mathrm{~m} / \mathrm{z}$ at the rate of 1.0 $\mathrm{scan} / \mathrm{s}$. Identification of different components in essential oil was based on the comparison with the known constituents that stored in National Institute of Standards and Technology Library (NIST14.0).

\section{Preparation and infrared spectroscopy (IR) characterization of polysaccharides from Renshen and Baizhu in SZC}

Renshen (200 g) or Baizhu (200 g) powder was refluxed by distilled water $(10 \times)$ for $2 \mathrm{~h}$. The procedure was repeated 3 times. Then these extracted solutions were combined, concentrated, and cooled $\left(4{ }^{\circ} \mathrm{C}\right)$ for $12 \mathrm{~h}$. The cooled concentrate solution was precipitated in $60 \%$ ethanol, and final residues were dried at $40^{\circ} \mathrm{C}$ in a vacuum oven. The characterization was carried out on a FTIR Spectrum (cary 660, Agilent, USA). Polysaccharides (1 mg) from Renshen or Baizhu were mixed with $150 \mathrm{mg} \mathrm{KBr}$ and homogenized by a pestle in the mortar. The mixture was then made by a hydraulic press into a slice, which was analyzed in IR at the wavenumber region from 400 to $4000 \mathrm{~cm}^{-1}$.

\section{Experimental design}

According to the clinical dosages of Renshen ( $3 \mathrm{~g}$ per time) and Baizhu (3g per time) (two Chinese medicines in SZC) in China Phannacopeia [22, 28, 29], we chose the oral doses of ginsenosides and polysaccharides from Renshen as $126.7 \mathrm{mg} / \mathrm{kg} / \mathrm{d}$ and $176.3 \mathrm{mg} / \mathrm{kg} / \mathrm{d}$ respectively, and the oral doses of essential oil and polysaccharides from Baizhu as $35.3 \mu \mathrm{L} / \mathrm{kg} / \mathrm{d}$ and $192.3 \mathrm{mg} / \mathrm{kg} / \mathrm{d}$ respectively for mice. SZC was mixed in $0.1 \%$ sodium carboxymethyl cellulose solution. Twenty-four mice were randomly assigned into three groups $(n=8)$ : the control, model and SZC groups, with 4 males and 4 females per group. Our modeling method was based on the existing literature [30]. The mice were orally administrated with SZC in a preventive mode on the first 2 days of the experiment, and then intraperitoneally injected with 5 -FU $(40 \mathrm{mg} / \mathrm{kg} / \mathrm{d})$ for 6 days. Mice in the SZC group were orally administrated with SZC half an hour before 5 -FU chemotherapy. SZC treatment lasted until the 3rd day after the end of 5-FU chemotherapy. Mice in the control group received equal volume of liquid solvent for the whole 11 days, while the untreated mice in the model group received 5-FU chemotherapy on the $3-8$ days. 
Both body weights and diarrhea states were recorded every day. For the diarrhea evaluation, the mice with moist and unformed stools, or perianal stains, were deemed to show the clinical symptoms of diarrhea. The calculation formula of diarrhea rates in each group was given as follow: the diarrhea rate $(\%)=$ the number of diarrheal mice/the number of mice $\times 100 \%$. Twenty-four hours after the last administration, fecal sample $(4 \mathrm{~g})$ was collected in sterile plastic tube and stored at $-80^{\circ} \mathrm{C}$. All the animals were sacrificed by cervical dislocation. Then thymuses and spleens were removed after the sacrifice of mice. Thymus and spleen indexes of each mouse were calculated according to the following formula: thymus (spleen) index $=$ thymus (spleen) weight/body weight $(\mathrm{mg} / \mathrm{g})$.

\section{Histological observation}

The colon segments $(2 \mathrm{~cm})$ of each mouse were collected and fixed in $4 \%$ formaldehyde solution after the sacrifice of mice. Then the colonic samples were embedded by paraffin, sliced $(4 \mu \mathrm{m})$, and stained with hematoxylin-eosin (HE). The histological changes of colonic segments were observed with the microscope (DMI300B, Leica, Germany).

\section{Gut microbiota analysis}

The genomic DNA of fecal samples was extracted by sodium dodecyl sulfate (SDS). After that, the concentration of DNA was detected using agarose gel electrophoresis. The V3-V4 region of bacterial 16S rRNA gene was amplified by polymerase chain reaction (PCR) with $341 \mathrm{~F}-$ 806R primer set. All PCR reactions were induced by High-Fidelity PCR Master Mix (New England Biolabs, USA). PCR products were detected by electrophoresis with 2\% agarose gel, and then purified by Qiagen Gel Extraction Kit (Qiagen, Germany). TruSeq ${ }^{\circ}$ DNA PCRFree Sample Preparation Kit (Illumina, USA) was used to generate sequencing library. Library quality was evaluated on Qubit@2.0 Fluorometer (Thermo Scientific, USA) and Agilent Bioanalyzer 2100 system. Finally, the Library was conducted on Illumina HiSeq 2500 platform (Novogene, Beijing, China).

\section{Bioinformatics analysis}

Paired-end reads of each sample were merged by FLASH (Version 1.2.7) to get raw tags. High-quality clean tags were generated from the strict filtration of raw tags by QIIME (Version 1.9.1). Final effective tags were produced after the removal of chimera sequences in the tags, which were detected by gold database (Version 1.9.1) and UCHIME Algorithm. The sequences at 97\% identity were clustered into the same operational taxonomic units (OTUs) by Uparse (Version 7.0.1001). Then the representative sequence of each OTU was selected for the species annotation. MUSCLE (Version 3.8.31) was used for the multiple sequence alignments to get the phylogenetic relationships. The OTUs abundance information was normalized by the sequence number standard. ACE and Shannon indexes that respectively indicated richness and diversity of gut microbiota were calculated on QIIME (Version 1.7.0). Linear discriminant analysis effect size (LEfSe) was used to detect the dominant bacterial community difference among groups. The lowest screening value of linear discriminant analysis (LDA) in LEfSe software was defaulted to 4. Venn, LEfSe and Non-Metric Multi-Dimensional Scaling (NMDS) diagrams were built on $\mathrm{R}$ software (Version 2.15.3). Unweighted Pair-group Method with Arithmetic Means (UPGMA) Clustering was performed on QIIME software (Version 1.7.0).

\section{Statistical analysis}

Statistical analysis was performed on SPSS (Version 21). Data were presented as means \pm Standard Error of Mean (SEM). One-way analysis of variance (ANOVA) followed by Least Significant Difference (LSD) test was used to analyze the statistical differences between multiple groups. Kruskal-Wallis test was applied if the data did not meet the assumptions of ANOVA. A value of $P<$ 0.05 was considered statistically significant.

\section{Results}

\section{Chemical compositions of SZC}

As shown in Table 1, the quantitations of ginsenosides standards showed good linearities within $6.25-200.00 \mu \mathrm{g} /$ $\mathrm{mL}$. The compositions of ginsenosides Rg1, Re, Rf, Rb1, $\mathrm{Rc}, \mathrm{Rb} 2$ and $\mathrm{Rd}$ in total ginsenosides were $46.98 \pm 0.25$, $55.25 \pm 0.66, \quad 12.81 \pm 0.11, \quad 132.81 \pm 0.96, \quad 83.86 \pm 0.39$, $70.66 \pm 0.34$, and $31.61 \pm 0.32 \mathrm{mg} / \mathrm{g}$, respectively (Fig. 1a). As shown in Fig. 1b, the five main constituents such as $\beta$ eudesmene (3.82\%), eudesma-3,7(11)-diene (12.49\%), atractylone (51.67\%), isopetasan (2.07\%), and 1-methoxy2-(1-methyl-2-methylenecyclopentyl) benzene (5.10\%) were identified in essential oil of Baizhu. Atractylone was

Table 1 Linear relationships with correlation coefficients for ginsenosides standards

\begin{tabular}{llll}
\hline Standards & Linear relationships & $r$ & Linear range $(\mu \mathrm{g} / \mathrm{mL})$ \\
\hline Rg1 & $Y=18.72 X+40.44$ & 0.9999 & $6.25-200.00$ \\
Re & $Y=14.48 X+40.82$ & 0.9995 & $6.25-200.00$ \\
Rf & $Y=20.58 X+55.34$ & 0.9993 & $6.25-200.00$ \\
Rb1 & $Y=13.99 X+47.49$ & 0.9995 & $6.25-200.00$ \\
Rc & $Y=14.30 X+25.17$ & 0.9998 & $6.25-200.00$ \\
Rb2 & $Y=13.79 X+13.62$ & 0.9999 & $6.25-200.00$ \\
Rd & $Y=16.78 X+25.37$ & 0.9995 & $6.25-200.00$ \\
\hline
\end{tabular}



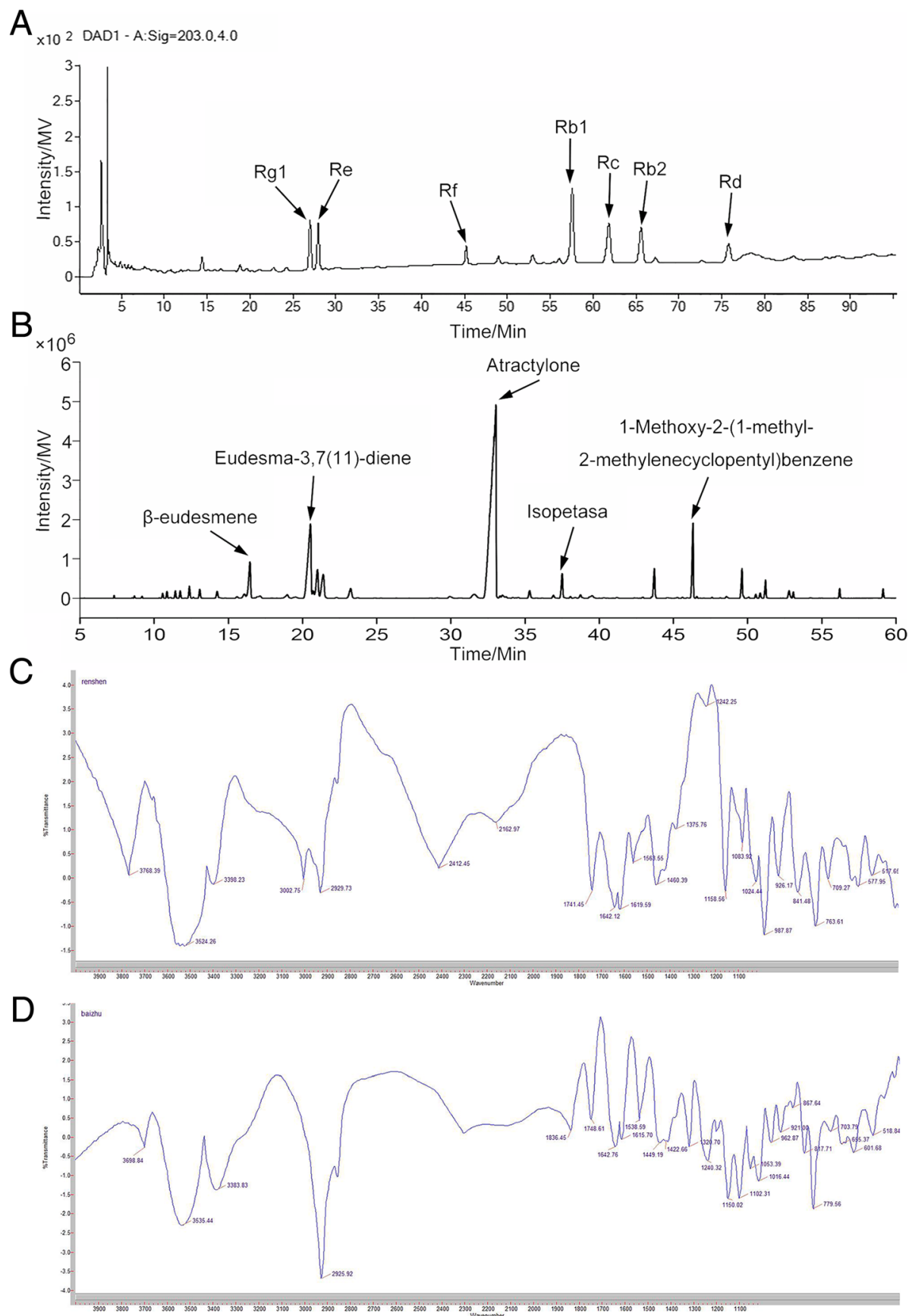

Fig. 1 Chemical characterization in SZC. (a) HPLC profile of ginsenosides from Renshen. (b) GC-MS profile of essential oil from Baizhu. (c) IR absorption spectrum of polysaccharides from Renshen. (d) IR absorption spectrum of polysaccharides from Baizhu

considered as the representative constituent of essential oil in Baizhu.

IR scanning results showed that both polysaccharides extracts of Renshen and Baizhu showed characteristic functional groups of polysaccharides (Fig. 1c and d). The bands at $3524.26 \mathrm{~cm}^{-1}$ and $3535.44 \mathrm{~cm}^{-1}$ were due to $\mathrm{O}-\mathrm{H}$ groups of polysaccharides from Renshen and Baizhu, respectively. The $\mathrm{C}=\mathrm{O}$ groups in
Renshen and Baizhu polysaccharides caused the increases of the absorption bands at $1741.45 \mathrm{~cm}^{-1}$ and $1748.61 \mathrm{~cm}^{-1}$, respectively. In addition, the bands at $1158.56 \mathrm{~cm}^{-1}$ and $1150.02 \mathrm{~cm}^{-1}$ (polysaccharides of Renshen and Baizhu, respectively), which were also the characteristic absorption bands of polysaccharides, resulted from the stretching vibration of $\mathrm{C}-\mathrm{O}$ in $\mathrm{C}-\mathrm{O}$ $\mathrm{C}$ cyclic ether. 
SZC ameliorates diarrhea and increases thymus/spleen indexes in mice with CID

The mice were injected with 5-FU for 6 days during the treatment of SZC (Fig. 2a). During the first 4 days, there was no significant difference in body weights among the control, model and SZC groups. Mice in the model group injected with 5-FU experienced weight loss compared with the control group from days 5-10, and had the lowest weight at the end of the experiment. Treatment with SZC significantly prevented weight loss in CID mice (Fig. 2b). From days 4-8, diarrhea rates increased in the untreated model group, while the clinical symptoms of diarrhea were not observed in the control group. At all time points, the diarrhea rates of the SZC group were obviously lower than those of the model group (Fig. 2c). In addition, the thymus and spleen indexes in the model group decreased significantly after 5FU chemotherapy, whereas SZC markedly inhibited the decreases of thymus and spleen indexes (Fig. 2d).

\section{SZC reduces colonic pathological changes in mice with CID}

HE-stained colonic section of the model group exhibited remarkable destructions of colonic mucosa and surface epithelia, which were not observed in the control group. Also, in the model group, goblet cells were obviously damaged by 5 -FU, and the numbers of goblet cells decreased significantly compared with the control group. However, these histological changes in colonic section were significantly reduced by SZC (Fig. 3).

\section{SZC modulates the overall structure of gut microbiota in mice with CID}

To detect structural differences in gut microbiota between the three groups, a total of 883,537 sequences and an average of 58,902 sequences per group were obtained from the 15 samples. Figure 4a showed that the position of box diagram in species accumulation boxplot tended to be gentle as the increase of samples number, which suggested that samples number and species richness both were adequate and stable. At this sequencing depth, the overall bacterial diversity had already been covered. Then the sequences were clustered into OTUs at $97 \%$ similarity, and 935 OTUs in total were obtained from 15 fecal samples. As shown in Fig. 4b, there were 424 OTUs in all three groups, and 17, 329 and 13 unique OTUs were detected in the control, model and SZC groups, respectively. Additionally, the model group showed a significant increase in the number of OTUs compared with the control group. But no significant difference of OTUs number existed between the control and SZC groups (Fig. 4c). Consistent with the changes of OTUs, both ACE richness and Shannon diversity values of bacterial community were significantly

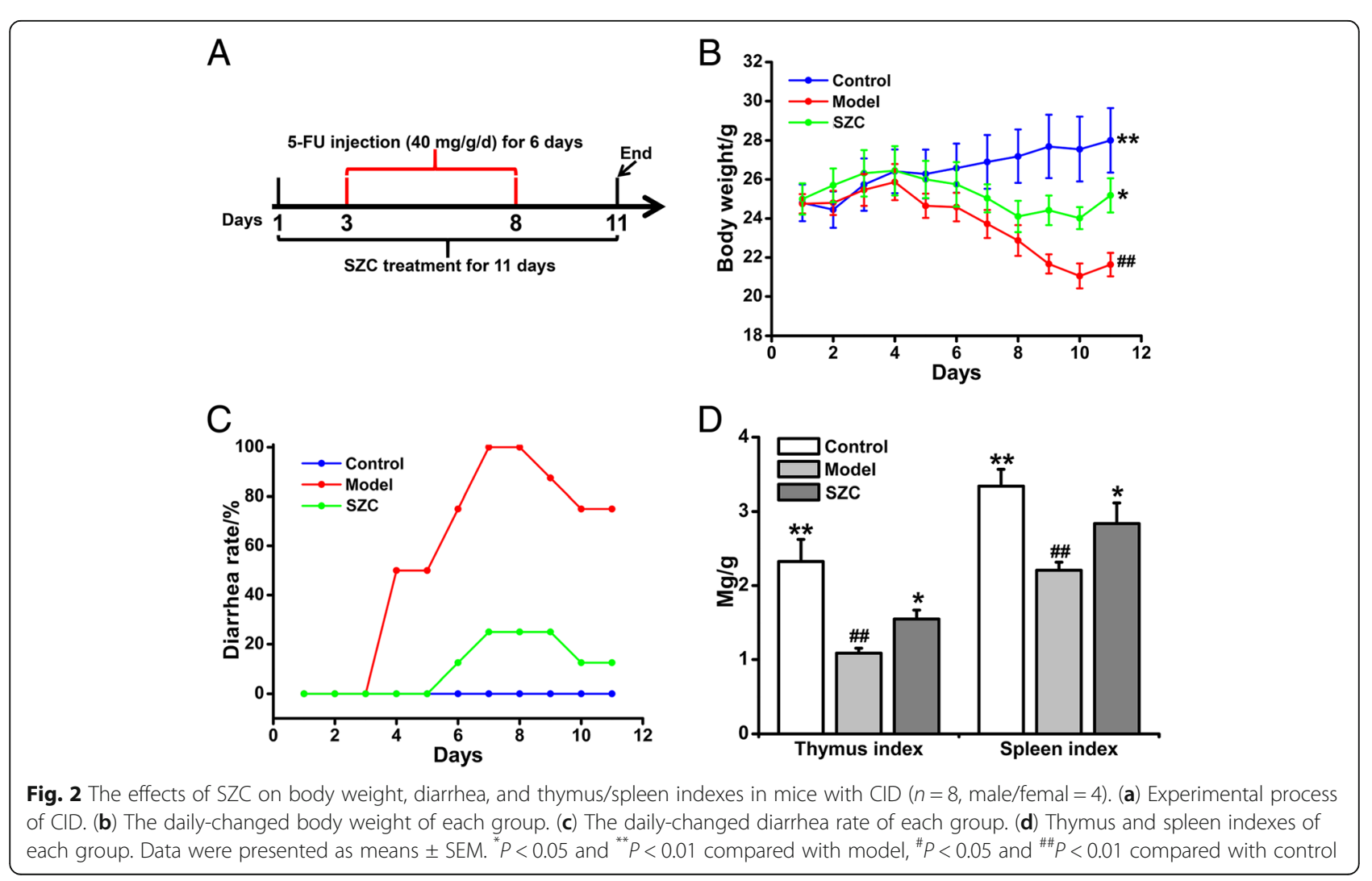




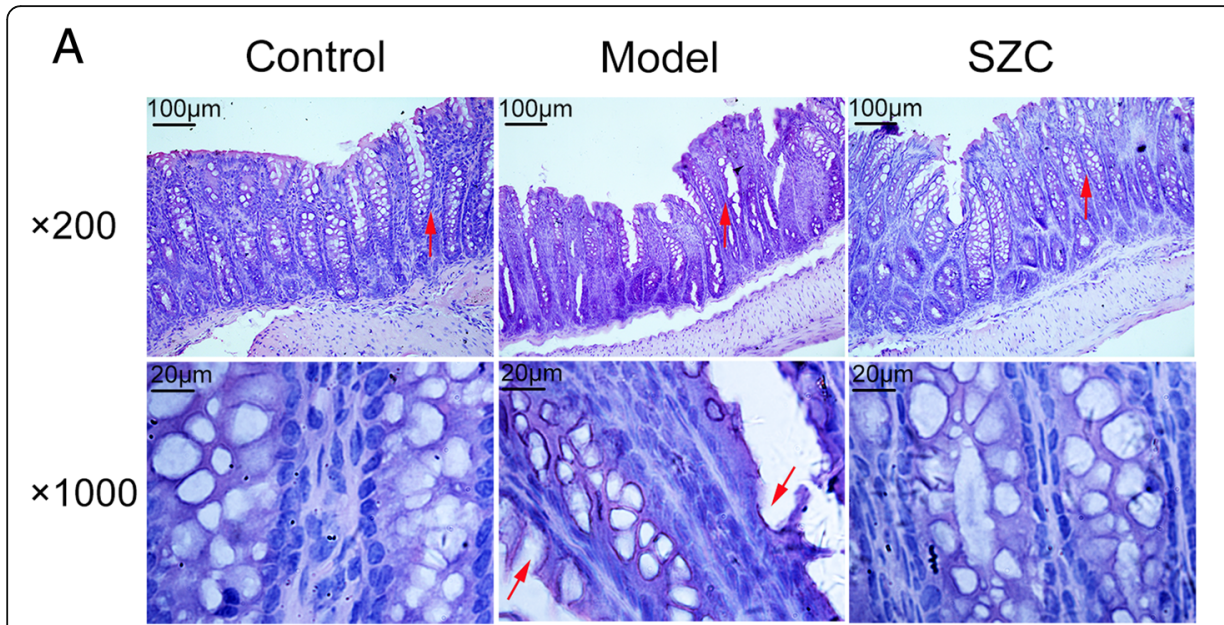

B

Fig. 3 The effects of SZC on histological changes of colon sections in mice with CID $(n=8$, male/femal =4). (a) Representative images of HEstained colonic sections ( $200 \times$ and $1000 \times$ magnifications). (b) Number of goblet cells in HE-stained colonic sections $(1000 \times$ magnification). Red arrows in colonic sections ( $200 \times$ magnification) indicated amplified target areas of pictures, and red arrows in colonic sections $(1000 \times$ magnification) indicated damaged goblet cells. Data were presented as means \pm SEM. ${ }^{*} P<0.05$ and ${ }^{* *} P<0.01$ compared with model, ${ }^{\#} P<0.05$ and ${ }^{\# \#} P<0.01$ compared with control

increased in the model group. After the treatment of SZC, ACE richness and Shannon diversity values reduced to the normal levels (Fig. $4 \mathrm{~d}$ and e). NMDS plot showed that the structure of overall bacterial community in the model group was quite different from those in the control and SZC groups (Fig. 4f). The clustering tree based on UPGMA also showed a significant separation in gut microbiota between the control and model groups. Notably, gut microbiota of mice treated with SZC did not significantly differ from that of the control group (Fig. 4g).

\section{SZC modulates the key phylotypes of gut microbiota in mice with CID}

The histogram of LDA value distribution showed that there were significant differences in dominant microbiota among the three groups (Fig. 5a). 1, 8 and 4 taxa of dominant communities were found in the control, model and SZC groups, respectively. Bacteroidales_S247_group was the dominant bacteria in the control group. The gut microbiota in the model group was dominated by Bacteroidetes, Bacteroidaceae, Prevotellaceae, Bacteroides and Alloprevotella. Dominant microbiota in the SZC group included Firmicutes and Clostridia. An evolutionary branch graph based on LDA scores further revealed the important microbiota in the three groups. The results showed that Bacteroidales_S24-7_group was identified as the dominant bacteria in the control group. In the model group, predominant microbiota consisted of Bacteroidaceae and Prevotellaceae. Lachnospiraceae, Clostridiales and Clostridia were the predominant flora in the SZC group (Fig. 5b).
Histograms with respect to phylum, family and genus were used to reveal the microbial species and their relative abundances (Fig. 6a-c). In fecal samples, Firmicutes, Bacteroidetes and Proteobacteria were the 3 dominate phyla, whose relative abundances were 77.62, 18.83 and $1.83 \%$ respectively in the control group. The abundances of Bacteroidetes (26.41\%) and Proteobacteria (8.76\%) increased in the model group, whereas the abundance of Firmicutes $(61.75 \%)$ decreased. However, SZC treatment could restore the proportions of Firmicutes (80.54\%), Bacteroidetes (11.74\%) and Proteobacteria (3.22\%) after 5-FU chemotherapy. At family level, our result showed that relative abundances of families such as Bacteroidaceae (8.19\%) and Prevotellaceae (8.03\%) significantly increased in the model group, compared with those (2.49 and $2.04 \%$, respectively) in the control group. Moreover, SZC treatment significantly reduced the abundances of Bacteroidaceae (2.53\%) and Prevotellaceae (2.87\%).

In addition, at genus level, the model group gave rise to high abundances of some potential pathogens such as Clostridiumm (0.50\%), Bacteroides (8.19\%), Parabacteroides (1.01\%), Alloprevotella (6.53\%), Desulfovibrio (0.73\%), Acinetobacter (0.40\%) and Pseudomonas (0.46\%). However, the relative abundances of these genera in the control group were significantly different, with Clostridiumm, Bacteroides, Parabacteroides, Alloprevotella, Desulfovibrio, Acinetobacter and Pseudomonas constituting 0.01, 2.49, 0.35, 1.66, $0.30,0.00 \%$ and 0 , respectively. In the SZC group, the inhibitory effects on Clostridiumm (0.10\%), Bacteroides (2.53\%), Parabacteroides (0.37\%), Alloprevotella (2.28\%), Desulfovibrio (0.48\%), Acinetobacter (0.00\%) and Pseudomonas $(0.00 \%)$ were observed compared with those in the untreated model group (Fig. 6d-f). 


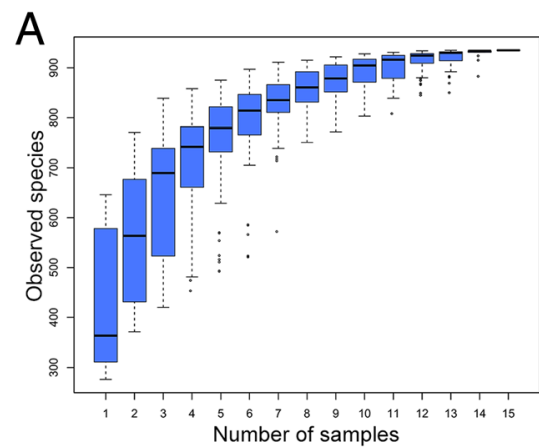

C

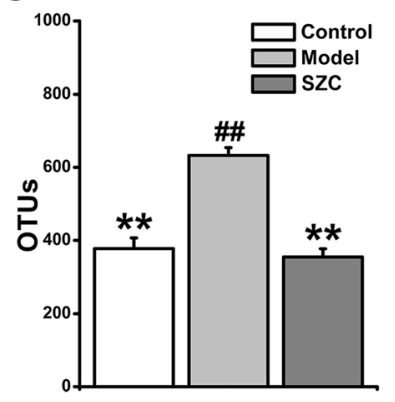

$\mathrm{F}$

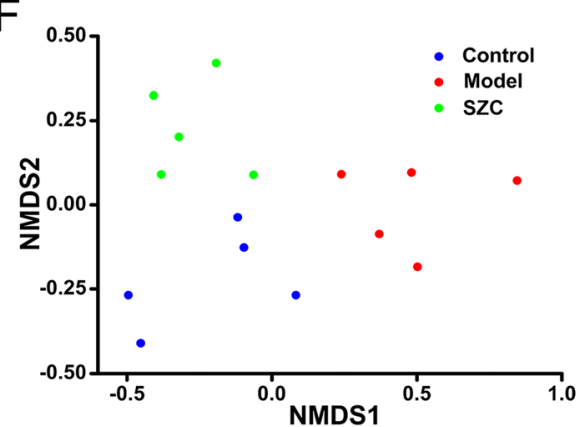

B

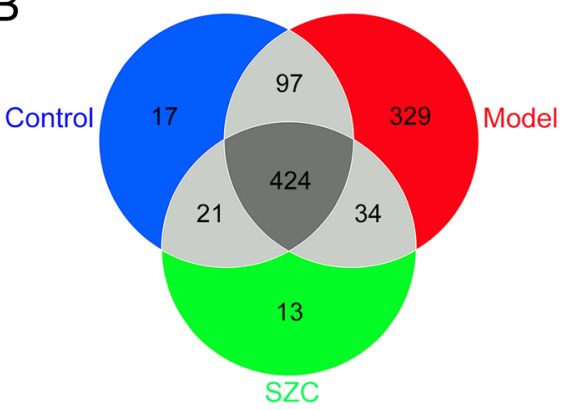

$\mathrm{E}$

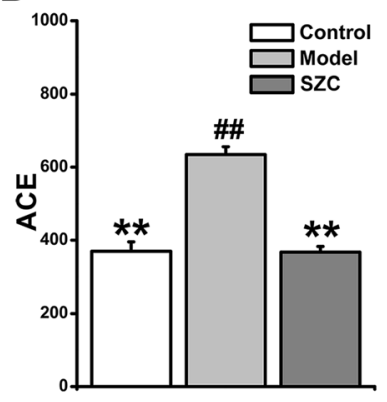

G

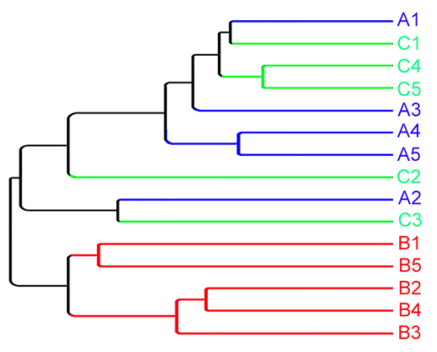

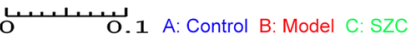

Fig. 4 The effects of SZC on the overall structure of gut microbiota in mice with CID ( $n=5$, male/femal =2-3). (a) Species accumulation boxplot was used for the estimations of samples number and species richness. (b) Venn diagram indicated the overlap of OTUs in the feces. (c) OTUs were clustered from high-quality sequences with $97 \%$ similarity. (d-e) ACE and Shannon values indicated the bacterial community richness and diversity, respectively. (f) NMDS plot showed distinct structural changes of the overall bacterial community in each group. (g) UPGMA clustering was used to interpret the distance matrix of each sample by the average linkage. Data were presented as means \pm SEM. ${ }^{*} P<0.05$ and ${ }^{* *} P<0.01$ compared with model, ${ }^{\#} P<0.05$ and ${ }^{\# \#} P<0.01$ compared with control

\section{Discussion}

In this study, all the model mice showed the clinical symptoms of diarrhea. We also found that thymus and spleen indexes in the model mice decreased significantly compared with those of the control group. Thymus and spleen indexes, whose levels depend on the extent of lymphocyte proliferation, can be used to partly reflect host immune function [31]. The results indicated that the immune system of the model group was affected by 5-Fu chemotherapy. In addition, some histological changes of colonic sections were observed in model mice. Especially, the numbers of goblet cells in the model group were remarkably lower than those of the control group. Goblet cells play important roles in producing and maintaining protective mucus blankets by synthesization and secretion of high molecular weight glycoprotein such as mucin. The reduction of goblet cell number could directly or indirectly impact intestinal health [32]. However, diarrhea and intestinal histological changes (Fig. 2) were significantly alleviated by SZC, which indicated that SZC was effective in the treatment of CID. More importantly, the potential mechanism behind this anti-diarrheal effect of SZC needed to be investigated in this study.

Previous studies have demonstrated that the pharmacological effects of Renshen and Baizhu were closely associated with gut microbiota. Renshen could effectively reduce body weight and change gut microbial 


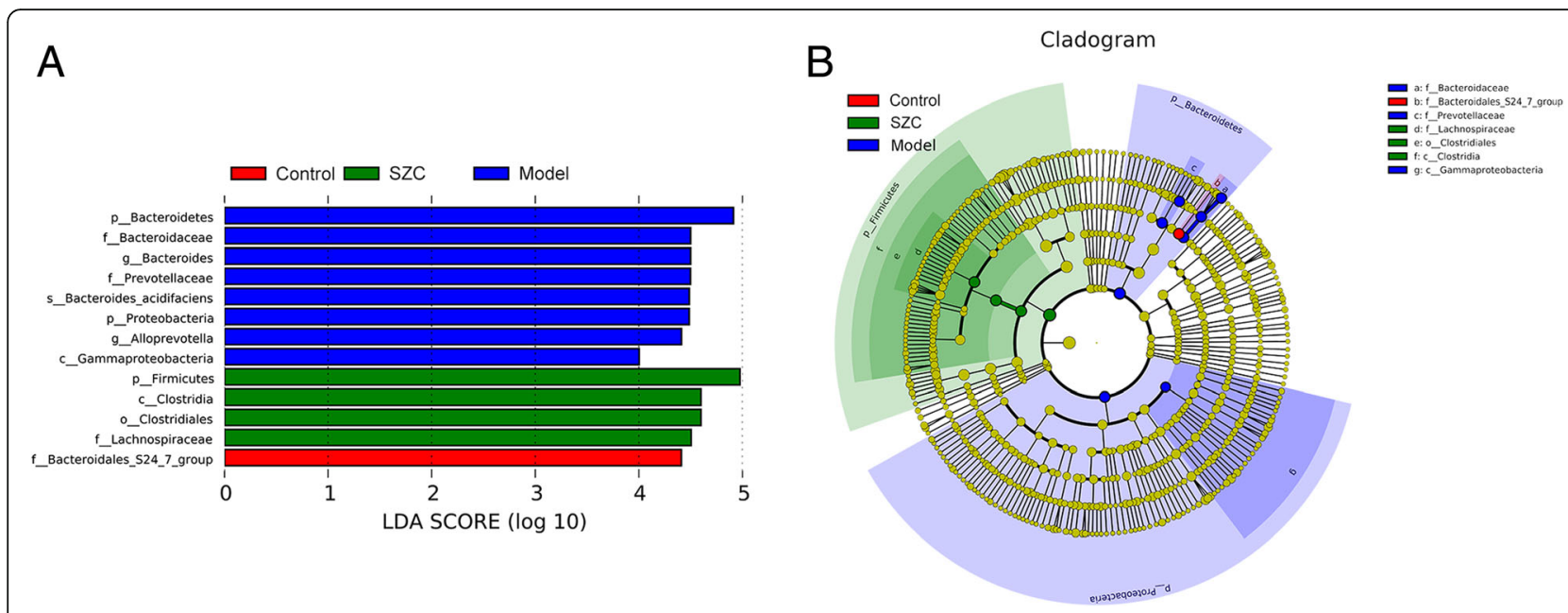

Fig. 5 Statistical difference in dominant microbiota among groups $(n=5$, male/femal $=2-3)$. (a) The histogram of LDA value distribution. (b) The evolutionary branch graph

composition in obese middle-aged Korean women [33]. And Baizhu was capable of reducing intestinal $\mathrm{pH}$ value, improving the adhesion of Bacillusbifidus, and fostering the normal gut microbiota in lincomycin-induced mice [34]. Therefore, SZC, a formula composed of active herbal extracts from Renshen and Baizhu, might exert potentially modulatory effects on gut microbiota. In addition, gut microbiota is a contributing factor of many diseases including diarrhea [35]. Therefore, we further analyzed gut microbiota from the feces of CID mice.

Gut microbial composition in fecal samples was determined by $16 \mathrm{~S}$ rDNA Amplicon Sequencing. Species accumulation boxplot indicated that the species richness of gut microbiota was sufficient and stable for the following analyses when the total number of samples was 15 ( $n=5$ in each group) (Fig. 4a). As shown in Fig. 4d and e, gut microbial richness and diversity were significantly increased in the model group. In general, the richness and diversity of microbial populations should be reduced under a pathological condition. The possible cause behind our results was that the richness and diversity of original microbiota was not reduced, and some potential pathogenic bacteria was introduced or increased in CID mice. Our results also showed that microbial richness and diversity decreased to normal levels after SZC treatment. Furthermore, the remarkable differences of distance existed among the three groups in NMDS plot, but a shorter distance was presented between the control and SZC groups. UPGMA clustering showed no obvious structural shift between the SZC and control groups, whereas the gut microbiota in the model group significantly deviated from that of the control group. Taken together, these results demonstrated the overall structure of gut microbiota in CID mice could be reversed to normal by SZC treatment.
Redundancy information uncovered the structural changes in the key phylotypes of gut microbiota based on the following classification: phylum, family and genus. Statistical results revealed that the abundances of Firmicutes and Proteobacteria significantly changed in the model group. But there was no difference between the SZC and control groups in the abundances of Firmicutes and Proteobacteria. Also, mice in the model group significantly increased the abundances of some genera such as Clostridiumm, Bacteroides, Parabacteroides, Alloprevotella, Desulfovibrio, Acinetobacter and Pseudomonas, which might provide opportunities for the occurrence of diarrhea. For example, high-abundant clostridium belonging to Firmicutes is one of the causes of diarrhea [36]. Bacteroides (Bacteroidaceae family and Bacteroidetes phylum), a complex organism colonized in the gut, may be the key factor influencing the integrity and immune development of intestinal mucosa in the process of diarrhea. Increased Bacteroides may also induce inflammation or even carcinogenesis [37, 38]. Parabacteroides in high abundance was found in immunerelated diarrhea [39]. In addition, Alloprevotella (Prevotellaceae family) is an anaerobic Gram-negative bacilli with the high relative abundance in patients with inflammatory bowel disease (IBD) [40]. Both Desulfovibrio and Acinetobacter (Proteobacteria family) were associated with bloody diarrhea [41, 42]. Pseudomonas is commonly found in patients with malnourished and infectious diarrhea [43, 44]. These above studies indicated that the growth of these genera (potential pathogens) was associated with diarrhea. As shown in Fig. 6, SZC treatment reduced the abundances of these genera in CID mice. Therefore, our results suggested that SZC could inhibit the growth of potential pathogens and modulated microbial structure in the feces. 


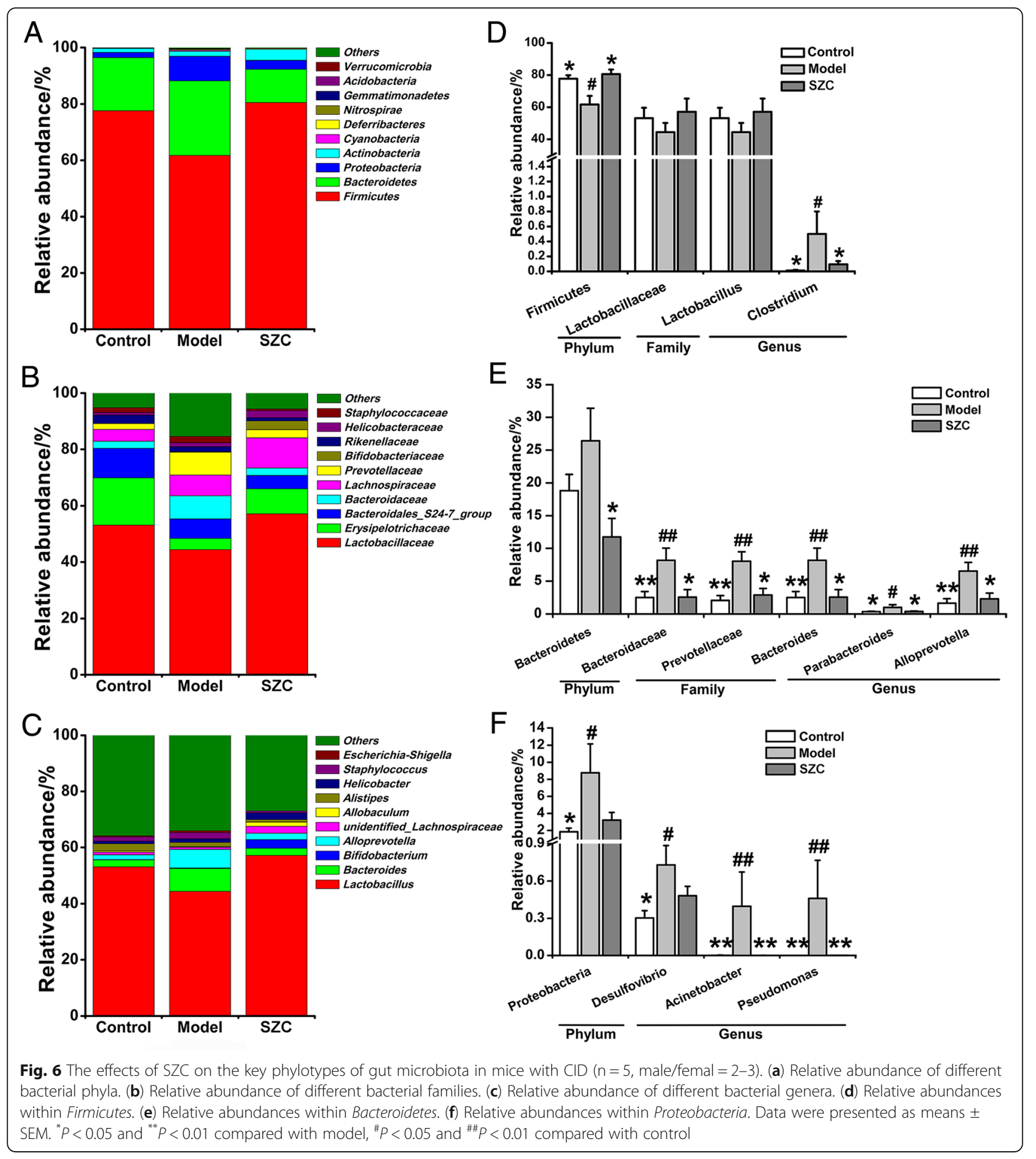

Moreover, Lactobacillus is an important genus for relieving diarrhea [45]. On the contrary, no significant difference in the abundances of Lactobacillus (probiotic) existed among the three groups, which was not in accordance with the results of 5-FU-induced decrease of Lactobacillus in the previous study [46]. The reason behind the difference might be that our results were based on fecal microbiota in mice, while the previous study focused on fecal microbiota in rats. Therefore, the relationship between Lactobacillus and CID still needs a deeper investigation. Conclusively, these results suggested that amelioration of CID with SZC treatment might be mainly mediated by inhibition of potential pathogens. 
Although SZC could modulate gut microbial structure of fecal samples in CID mice, the specific relationship between the anti-diarrheal activity of SZC and gut microbiota remained undefined. For example, fecal collection in a non-invasive manner was chosen as the sampling method for gut microbial analysis. However, fecal communities may not completely represent bacterial communities living in intestines, although they play an important role in maintaining intestinal homeostasis and microbial diversity [47, 48]. In addition to structural modulation of gut microbiota, the therapeutic effects of herbal drugs could also be influenced by gut microbial metabolism and pharmacokinetics [49]. Therefore, there is still no systematic and comprehensive interpretation for the association between the anti-diarrheal effect of SZC and gut microbiota, which will be our focus in the future researches.

\section{Conclusion}

In conclusion, our study found that gut microbial structure was modulated by SZC (the Chinese herbal formula) during the alleviation of CID. Especially, at genus level, SZC significantly inhibited the growth of potential pathogens closely related to diarrhea, including Clostridiumm, Bacteroides, Parabacteroides, Alloprevotella, Acinetobacter and Pseudomonas. These results indicated that gut microbial modulation was associated with the anti-diarrheal activity of SZC.

\section{Abbreviations \\ 5-FU: 5-Fluorouracil; ANOVA: Analysis of variance; CDUTCM: Chengdu University of Traditional Chinese Medicine; CID: Chemotherapy-induced diarrhea; GC-MS: Gas chromatography-mass spectrometer; \\ Gl: Gastrointestinal; HE: Hematoxylin-eosin; HPLC: High performance liquid chromatography; IBD: Inflammatory bowel disease; IR: Infrared spectroscopy; LDA: Linear discriminant analysis; LEfSe: Linear discriminant analysis effect size; LSD: Least Significant Difference; NMDS: Non-Metric Multi-Dimensional Scaling; OTUs: Operational taxonomic units; PCR: Polymerase chain reaction; SDS: Sodium dodecyl sulfate; SEM: Standard Error of Mean; SZC: Shenzhu Capsule; UC: Ulcerative colitis; UPGMA: Unweighted Pair-group Method with Arithmetic Means}

\section{Acknowledgments}

Not applicable.

\section{Authors' contributions}

JW and WWF performed the experiments, analyzed data and wrote the manuscript. SYZ, FT and YCS contributed to data analysis and technical graphics. LC contributed to the identification of medicinal materials. HA designed the experiment and wrote the manuscript. CP supported and supervised the study. All authors read and approved the final manuscript.

\section{Funding}

This study was supported by National Natural Science Foundation of China (No. 81891012, 81630101, and 81503272) and Department of Science and Technology of Sichuan Province (NO. 2017JY0187 and 2017TD0001).

\section{Availability of data and materials}

The data supporting this study are available from the corresponding author upon reasonable request.

\section{Ethics approval and consent to participate}

All experimental procedures involving animals were conformed to the strict standards of Animal Ethics Committee (Approval No. AEC-201706) in CDUTCM.

\section{Consent for publication}

Not applicable.

\section{Competing interests}

All the authors declared there was no conflict of interest

\section{Author details}

${ }^{1}$ School of Pharmacy, Chengdu University of Traditional Chinese Medicine, Chengdu 611137, China. ${ }^{2}$ Innovative Institute of Chinese Medicine and Pharmacy, Chengdu University of Traditional Chinese Medicine, Chengdu 611137, China

Received: 19 December 2018 Accepted: 3 June 2019

Published online: 11 June 2019

\section{References}

1. Lozupone CA, Stombaugh JI, Gordon Jl, Jansson JK, Knight R. Diversity, stability and resilience of the human gut microbiota. Nature. 2012;489(7415): 220-30.

2. Flint HJ, Scott KP, Louis P, Duncan SH. The role of the gut microbiota in nutrition and health. Nat Rev Gastroenterol Hepatol. 2012;9(10):577-89.

3. Jandhyala SM, Talukdar R, Subramanyam C, Vuyyuru H, Sasikala M, Reddy DN. Role of the normal gut microbiota. World J Gastroenterol. 2015;21(29): 8787-803.

4. Carroll IM, Ringel-Kulka T, Siddle JP, Ringel Y. Alterations in composition and diversity of the intestinal microbiota in patients with diarrhea-predominant irritable bowel syndrome. Neurogastroenterol Motil. 2012;24(6):521-30.

5. Frank DN, St Amand AL, Feldman RA, Boedeker EC, Harpaz N, Pace NR. Molecular-phylogenetic characterization of microbial community imbalances in human inflammatory bowel diseases. Proc Natl Acad Sci U S A. 2007:104(34):13780-5.

6. Tremaroli V, Bäckhed F. Functional interactions between the gut microbiota and host metabolism. Nature. 2012;489(7415):242-9.

7. Stock J. Gut microbiota: an environmental risk factor for cardiovascular disease. Atherosclerosis. 2013:229(2):440-2.

8. Jia W, Li H, Zhao L, Nicholson JK. Gut microbiota: a potential new territory for drug targeting. Nat Rev Drug Discov. 2008;7(2):123-9.

9. Faith JJ, Guruge JL, Charbonneau M, Subramanian S, Seedorf H, Goodman $\mathrm{AL}$, et al. The long-term stability of the human gut microbiota. Science. 2013:341(6141):1237439.

10. Kornblau S, Benson AB, Catalano R, Engelking C, Field M, Ippoliti C, et al. Management of cancer treatment-related diarrhea. Issues and therapeutic strategies J Pain Symptom Manage. 2000;19(2):118-29.

11. Stein A, Voigt W, Jordan K. Chemotherapy-induced diarrhea: pathophysiology, frequency and guideline-based management. Ther Adv Med Oncol. 2010;2(1):51-63.

12. lacovelli R, Pietrantonio F, Palazzo A, Maggi C, Ricchini F, de Braud F, et al. Incidence and relative risk of grade 3 and 4 diarrhoea in patients treated with capecitabine or 5-fluorouracil: a meta-analysis of published trials. $\mathrm{Br}$ J Clin Pharmacol. 2014;78(6):1228-37

13. Richardson G, Dobish R. Chemotherapy induced diarrhea. J Oncol Pharm Pract. 2007;13(4):181-98.

14. Gibson RJ, Keefe DM. Cancer chemotherapy-induced diarrhoea and constipation: mechanisms of damage and prevention strategies. Support Care Cancer. 2006;14(9):890-900.

15. Andreyev J, Ross P, Donnellan C, Lennan E, Leonard P, Waters C, et al. Guidance on the management of diarrhoea during cancer chemotherapy. Lancet Oncol. 2014;15(10):e447-60.

16. Zachariah B, Gwede CK, James J, Ajani J, Chin LJ, Donath D, et al. Octreotide acetate in prevention of chemoradiation-induced diarrhea in anorectal cancer: randomized RTOG trial 0315. J Natl Cancer Inst. 2010;102(8):547-56.

17. Kato S, Hayashi S, Kitahara Y, Nagasawa K, Aono H, Shibata J, et al. Saireito (TJ-114), a Japanese traditional herbal medicine, reduces 5-fluorouracilinduced intestinal mucositis in mice by inhibiting cytokine-mediated apoptosis in intestinal crypt cells. PLoS One. 2015;10(1):e0116213. 
18. Qi F, Zhao L, Zhou A, Zhang B, Li A, Wang Z, et al. The advantages of using traditional Chinese medicine as an adjunctive therapy in the whole course of cancer treatment instead of only terminal stage of cancer. Biosci Trends. 2015;9(1):16-34

19. Ao H, Peng C, Deng QX. Effect of Shenzhu capsules on upstream genes in mitochondrial pathway of cell apoptosis in gastric cancer mice with spleendeficience. Chin Pharmacol Bulletin. 2013;29(7):1033-4.

20. Ao H, Peng C, Deng QX. Effect of Shenzhu capsules on downstream genes in mitochondrial pathway of cell apoptosis in gastric cancer mice with spleen-deficience. Chin Pharmacol Bulletin. 2013;29(6):887-8.

21. Ao H, Peng C, Deng QX. Effect of Shenzhu capsules on the microstructure and ultrastructure of gastric cancer MKN45 cells. Pharmacology and Clinics of Chinese Materia Medica. 2013;29(2):149-52.

22. Ao $\mathrm{H}$, Peng $\mathrm{C}$, Lin $\mathrm{DH}$, Zhang $\mathrm{H}$, Ye B. Study on gene expression profile induced by the model with spleen-deficiency and gastric cancerometastasis in mice treated by Shenzhu Capules. J Yunnan Coll Tradit Chin Med. 2010;33(1):7-11.

23. Feng WW, Ao H, Yue SJ, Peng C. Systems pharmacology reveals the unique mechanism features of Shenzhu capsule for treatment of ulcerative colitis in comparison with synthetic drugs. Sci Rep. 2018;8(1):16160.

24. Bai YR, Dai EQ, Gao ZH, Liu W, Chen F, Wang XY. Clinical observation of Shenling Baizhu powder improving post-chemotherapy patients with rectal excision. Chinese Traditional Patent Medicine. 2017;39(2):278-82.

25. Gou H, Gu LY, Shang BZ, Xiong Y, Wang C. Protective effect of Bu-Zhong-YiQi decoction, the water extract of Chinese traditional herbal medicine, on 5-fluorouracil-induced intestinal mucositis in mice. Hum Exp Toxicol. 2016; 35(12):1243-51.

26. Chen JM, Yang TT, Cheng TS, Hsiao TF, Chang PMH, Leu JY, et al. Modified Sijunzi decoction can alleviate cisplatin-induced toxicity and prolong the survival time of cachectic mice by recovering muscle atrophy. J Ethnopharmacol. 2019;233:47-55.

27. Feng WW, Ao H, Peng C. Gut microbiota, short-chain fatty acids, and herbal medicines. Front Pharmacol. 2018;9:1354.

28. Chinese Pharmacopoeia Commission. Pharmacopoeia of the People's Republic of China. China Medical Science Press. 2015;1:8-9.

29. Chinese Pharmacopoeia Commission. Pharmacopoeia of the People's Republic of China. China Medical Science Press. 2015;1:103-4.

30. Li YF, Zhang BY, Lai Y, Chen ZJ. Effect of Banxia Xiexin tang on intestinal immune function in fluorouracil-induced diarrhea mice. Chin J Exp Tradit Med Form. 2014:20(23):180-4.

31. Yuan CF, Huang XN, Cheng L, Bu YQ, Liu GL, Yi FP, et al. Evaluation of antioxidant and immune activity of Phellinus ribis glucan in mice. Food Chem. 2009;115(2):581-4

32. Specian RD, Oliver MG. Functional biology of intestinal goblet cells. Am J Phys. 1991;260(2 Pt 1):C183-93.

33. Song MY, Kim BS, Kim H. Influence of Panax ginseng on obesity and gut microbiota in obese middle-aged Korean women. J Ginseng Res. 2014;38(2): $106-15$.

34. Cao JM, Yang XJ, Zhang WZ. Study on the effect and mechanism of Chinese herbs on intestinal normal flora of mice. Chin Arch Tradit Chin Med. 2012;30(2):393-5.

35. Sekirov I, Russell SL, Antunes LCM, Finlay BB. Gut microbiota in health and disease. Physiol Rev. 2010;90(3):859-904.

36. Polage CR, Solnick JV, Cohen SH. Nosocomial diarrhea: evaluation and treatment of causes other than Clostridium difficile. Clin Infect Dis. 2012; 55(7):982-9.

37. Sears CL, Islam S, Saha A, Arjumand M, Alam NH, Faruque AS, et al. Association of enterotoxigenic Bacteroides fragilis infection with inflammatory diarrhea. Clin Infect Dis. 2008:47(6):797-803.

38. Wick EC, Sears CL. Bacteroides spp. and diarrhea. Curr Opin Infect Dis. 2010; 23(5):470-4.

39. Liu T, Hu Y. Intestinal microbiota to predict risk for immune-related diarrhea in patients with lung cancer patients. J Clin Oncol. 2018:132.

40. Kiely CJ, Pavli P, O'Brien CL. 2018. The microbiome of translocated bacterial populations in patients with and without inflammatory bowel disease. Intern Med J. 2018;48(11):1346-54.

41. Polanco N, Manzi L. Toxigenic effect of Acinetobacter baumannii isolated from children with acute diarrhoea. Investig Clin. 2008;49(1):59-67.

42. Kushkevych IV. Dissimilatory sulfate reduction by various Desulfovibrio sp. strains of the human intestine. Мікробіологія і біотехнологія. 2013:3:50-63.
43. Omoike IU, Abiodun PO. Upper small intestinal microflora in diarrhea and malnutrition in Nigerian children. J Pediatr Gastroenterol Nutr. 1989;9(3): 314-21.

44. Porco FV, Visconte EB. Pseudomonas aeruginosa as a cause of infectious diarrhea successfully treated with oral ciprofloxacin. Ann Pharmacother. 1995;29(11):1122-3.

45. Guandalini S. Probiotics for prevention and treatment of diarrhea. J Clin Gastroenterol. 2011:45:S149-53.

46. Zhang T, Lu SH, Bi Q, Liang L, Wang YF, Yang XX, et al. Volatile oil from Amomi Fructus attenuates 5-fluorouracil-induced intestinal mucositis. Front Pharmacol. 2017:8:786.

47. Eckburg PB, Bik EM, Bernstein CN, Purdom E, Dethlefsen L, Sargent M, et al. Diversity of the human intestinal microbial flora. Science. 2005;308(5728): 1635-8.

48. Liu S, da Cunha AP, Rezende RM, Cialic R, Wei ZY, Bry L, et al. The host shapes the gut microbiota via fecal microRNA. Cell Host Microbe. 2016; 19(1):32-43.

49. Feng WW, Ao H, Peng C, Yan D. Gut microbiota, a new frontier to understand traditional Chinese medicines. Pharmacol Res. 2019;142:176-91.

\section{Publisher's Note}

Springer Nature remains neutral with regard to jurisdictional claims in published maps and institutional affiliations.
Ready to submit your research? Choose BMC and benefit from:

- fast, convenient online submission

- thorough peer review by experienced researchers in your field

- rapid publication on acceptance

- support for research data, including large and complex data types

- gold Open Access which fosters wider collaboration and increased citations

- maximum visibility for your research: over $100 \mathrm{M}$ website views per year

At BMC, research is always in progress.

Learn more biomedcentral.com/submissions 